\title{
(6) OPEN ACCESS \\ International patterns of dual antiplatelet therapy duration after acute coronary syndromes
}

\author{
Héctor Bueno, ${ }^{1,2,3}$ Stuart Pocock, ${ }^{4}$ Nicolas Danchin, ${ }^{5}$ Lieven Annemans, ${ }^{6}$ \\ John Gregson, ${ }^{4}$ Jesús Medina, ${ }^{7}$ Frans Van de Werf ${ }^{8}$
}

\begin{abstract}
- Additional material is
published online only. To view please visit the journal online (http://dx.doi.org/10.1136/ heartjnl-2016-309509).

${ }^{1}$ Centro Nacional de Investigaciones Cardiovasculares (CNIC), Madrid, Spain

${ }^{2}$ Cardiology Department, Hospital Universitario 12 de Octubre, and Instituto de investigación i+12

${ }^{3}$ Universidad Complutense de Madrid, Spain

${ }^{4}$ London School of Hygiene and Tropical Medicine, London, UK

${ }^{5}$ Hôpital Européen Georges Pompidou \& René Descartes University, Paris, France

${ }^{6}$-CHER Interuniversity Centre for Health Economics Research UGent, VUB, Ghent, Belgium

${ }^{7}$ Medical Evidence and Observational Research, Global Medical Affairs, AstraZeneca, Madrid, Spain

${ }^{8}$ University Hospitals, Leuven, Belgium
\end{abstract}

\section{Correspondence to} Dr Héctor Bueno, Centro Nacional de Investigaciones Cardiovasculares (CNIC) Melchor Fernandez Almagro, 3, Madrid 28029, Spain; hbueno@cnic.es

Received 18 February 2016 Revised 5 July 2016 Accepted 13 July 2016 Published Online First 8 August 2016

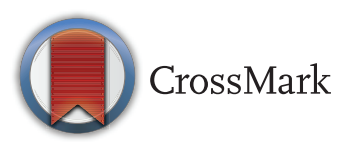

To cite: Bueno $\mathrm{H}$, Pocock $\mathrm{S}$, Danchin N, et al. Heart 2017:103:132-138.
ABSTRACT
Objective To describe international patterns of dua antiplatelet therapy (DAPT) duration after acute coronary syndrome (ACS), and explore its determinants and correlation with clinical events.

Methods EPICOR (long-tErm follow-uP of antithrombotic management patterns In acute CORonary syndrome patients) is a prospective, international, observational study of 10568 ACS hospital survivors enrolled in 555 centres from 20 countries across Europe and Latin America between 2010 and 2011, with telephone follow-up at quarterly intervals up to 24 months to assess treatment continuation and clinical events.

Results Of 8593 patients discharged on DAPT, 4859 $(57 \%)$ remained on uninterrupted DAPT at end of follow-up. There were minor differences in rates of DAPT discontinuation according to age, gender, risk factors, therapeutic strategy or region, but major differences between countries. By study end, 555 of evaluable patients $(5.7 \%)$ died, $727(10.0 \%)$ experienced new cardiovascular (CV) events, 496 new coronary events $(6.82 \%)$ and $154(2.11 \%)$ clinically relevant bleeding (14 (6.7\%) fatal). Most CV events and deaths (85\%) occurred while on DAPT. DAPT interruption was associated with increased risk of CV events in the following week (HR 2.29; 95\% Cl 1.08 to 4.84) but not specifically with time to first coronary event or mortality. Conclusions Despite guideline recommendations, most patients with ACS in Europe and Latin America remained on DAPT beyond 12 months, country being the most important determinant of DAPT duration. Increase in short-term CV risk was seen after switching from DAPT to less medication, as compared with continued DAPT, with no long-term effect on coronary or mortality risk. Trial registration number NCT01171404; Results.

\section{INTRODUCTION}

Recent evidence shows that prolonging dual antiplatelet therapy (DAPT) in patients treated with coronary stents for longer than 12 months reduces the rate of ischaemic events but increases bleeding events. ${ }^{1}$ Although duration of DAPT for 12 months following an acute coronary syndrome (ACS) was an arbitrary period, selected on the basis of duration of follow-up in the Clopidogrel in Unstable angina to prevent Recurrent Events (CURE) trial and findings from two other major DAPT trials in $\mathrm{ACS},{ }^{2-4}$ European and US ACS guidelines recommended the use of DAPT for up to 12 months for the majority of patients, treated with or without stents. ${ }^{5} 6$ Despite this uniform recommendation, information on DAPT duration from real-life practice is scarce, with some studies reporting observations from only a limited number of countries. ${ }^{7-9}$

The EPICOR (long-tErm follow-uP of antithrombotic management patterns In acute CORonary syndrome patients) study (NCT01171404) was designed to describe real-life international patterns of DAPT duration in patients with ACS. Secondary objectives were identifying determinants for different DAPT duration patterns and exploring the association of changes in DAPT use with cardiovascular (CV) or bleeding risk events. ${ }^{10}$

\section{METHODS}

\section{Study design}

Full details of the study design have been published previously. ${ }^{10}$ Briefly, EPICOR is a prospective, 'reallife', international, observational, cohort study, which enrolled 10568 consecutive survivors of an ACS between September 2010 and March 2011 from 555 hospitals in 20 countries across Europe and Latin America. Patients were enrolled at discharge from hospital but details of their management were recorded during the prehospital and inhospital periods as well as during follow-up (by 3 -monthly telephone interview) for up to 2 years from discharge.

\section{Study population}

The main inclusion criteria were: hospitalisation within $24 \mathrm{~h}$ of symptom onset of the index event and a final diagnosis of ST-segment elevation myocardial infarction (STEMI) or non-ST-segment elevation ACS (comprising both non-STEMI and unstable angina) at discharge, age $\geq 18$ years, and written informed consent. Patients were excluded if they had a 'secondary' ACS (precipitated by or occurring as a complication of surgery, trauma, gastrointestinal bleeding or percutaneous coronary intervention (PCI), or occurring during hospitalisation for other reasons). Other exclusion criteria included any condition/circumstance considered likely to limit completion of follow-up, any serious/ severe comorbidities limiting life expectancy to $<6$ months, or previous enrolment in EPICOR or another clinical trial. All other details (eg, site selection, patient enrolment and secondary objectives) are provided in the design manuscript. ${ }^{10}$ Patient baseline characteristics, and prehospital and inhospital management have also been described elsewhere. $^{11}$ 


\section{Follow-up and event definition}

Patients were followed up by centralised telephone interviews by well trained native speakers of each patient's language, and interviewers were supervised by a direct patient contact manager at 6 weeks after the index event, and then every 3 months up to 24 months. Interviews included questions related to the occurrence of events, ischaemic and/or bleeding; planned and unplanned hospitalisations, interventions or visits to the emergency room or other physicians (including dentists); treatment changes, including any planned/unplanned treatment interruptions; other healthcare resource utilisation; and quality of life measurement. For any changes in treatment, each drug of interest was listed in turn - aspirin, ticlopidine, clopidogrel, prasugrel, warfarin/acenocoumarol, dabigatran or any other to be specified - and patients were asked to provide details of when and why a current drug dose was changed, stopped and/ or restarted, or any new drug initiated. Patients were asked to specify if the treatment change was due to a hospitalisation or other physician visit, or at their own initiative. Events were recorded through specific questionnaires in which hospitalisations or emergency department visits were first recorded. In such cases, interviewers were asked to collect all relevant clinical information (medical reports) from the patient, hospital physicians or general practitioners. Whenever necessary, the primary study investigator was contacted to obtain confirmation or any clarification regarding the identified event.

The main reason for hospitalisation/visit was registered as CV, bleeding or other. All CV and bleeding events reported by patients, relatives or physicians were recorded together with specific information regarding each particular event but only events in which a medical record with a specific diagnosis was available were counted. Therefore, event rates were calculated according to diagnoses reported in medical records.

\section{Statistical analysis}

Three groups were defined for antithrombotic patterns according to patients' discharge medication: (1) DAPT, comprising patients discharged on aspirin plus one other oral antiplatelet agent, such as clopidogrel, prasugrel or ticlopidine (ticagrelor was not available in the majority of countries while the study was conducted); (2) oral anticoagulation, participants discharged on warfarin or dabigatran regardless of any other antithrombotic drug prescribed, and (3) single antiplatelet therapy (SAPT), which included all other patients. Characteristics across discharge medication groups were compared using the $\chi^{2}$ test. Event rates were calculated using the Kaplan-Meier method, and Cox proportional hazards models were used to relate change in DAPT to CV events. We did not use recurrent events for the present analysis. Follow-up of each patient was divided into time spent before and after change in DAPT. Change from DAPT was entered in the model as a time-updated categorical variable that enters the model on the day of change from DAPT. ${ }^{12}$ HRs therefore compare the hazard of an event after a change in DAPT to the hazard before a change in DAPT (which includes the hazard in patients who do not change from DAPT at any time). Time after change from DAPT was further split into $<7$ days, 8-30 days and $>30$ days after the change. All analyses were performed in Stata V.11.2 (StataCorp, USA, 2009).

\section{RESULTS}

\section{Patient characteristics}

Of the 10568 patients enrolled in EPICOR, 10069 had known medication status at discharge. Nineteen patients $(0.18 \%)$ were lost to follow-up, and 743 (7.03\%) discontinued due to voluntary withdrawal or enrolment in another clinical trial during follow-up. Of the 10069 patients, most $(8593,85.3 \%)$ were discharged on DAPT, $899(8.9 \%)$ on SAPT and $537(5.3 \%)$ on oral anticoagulation (with or without antiplatelet agents) (table 1). Forty patients were discharged on neither antiplatelets nor anticoagulants. Baseline characteristics, inhospital procedures and medications and discharge therapies for the overall population and the DAPT group have been described in detail elsewhere. ${ }^{11}$ There were small differences in the proportions of patients discharged on each type of antithrombotic option across patient characteristics except for age, initial management strategy and type of ACS.

\section{Change in medication}

Complete follow-up with full information on antiplatelet treatment was available for 8593 patients discharged on DAPT. A large proportion of patients discharged on DAPT did not change to another antiplatelet option at any time during the first 12 months $(6552,76.2 \%)$ and up to the end of follow-up $(4859,56.5 \%)$ (figure 1). In terms of specific antiplatelet treatment, there was no difference at 12 months, but at 23 months those on aspirin plus prasugrel were significantly more likely to have been switched $(\mathrm{p}<0.0001)$. The most common reasons for change of medication in patients on DAPT were instruction from a physician $(53.8 \%)$, programmed interventions (24.9\%) and physician instructions at discharge (9.6\%). Older patients, those with diabetes and those in Latin America or northern Europe were significantly more likely to remain on DAPT, and there was a borderline association with higher baseline haemoglobin level (table 2). There were statistically significant, although numerically minor, differences among regions in the proportion of patients remaining on DAPT at the end of follow-up, but major differences between countries were observed within regions $(\mathrm{p}<0.001$ for each region, figure 2$)$.

\section{Clinical events}

By the end of follow-up, 559 (5.7\%) patients had died, and 978 patients (9.8\%) experienced $1173 \mathrm{CV}$ events, of which 653 were coronary, including 252 myocardial infarctions (MIs) (table 3, see online supplementary figure). 'Non-coronary' events included congestive heart failure, arrhythmias and cerebrovascular events. A relatively small number of patients, $168(1.7 \%)$, had one or more clinically relevant bleeding events during follow-up, of which 14 ( $0.1 \%$ of study population) were fatal (table 3 , see online supplementary figure).

Most events and deaths (85\%) during follow-up occurred while patients were on DAPT. During the overall follow-up period, there was no apparent association between medication change and coronary or overall CV events (see online supplementary table), but there was an increased rate of $\mathrm{CV}$ events within the first 7 days after changing from DAPT to less medication (HR 2.29; 95\% CI 1.08 to 4.84) (figure 3). Non-fatal CV events within the first 7 days included two cases of unstable angina, and one case each of congestive heart failure, stroke, STEMI and ventricular arrhythmia. During that period, the incidence of any CV event was 9.2 per 100 person-years at risk compared with 5.9 during time on DAPT (HR 2.22; 95\% CI 1.30 to 3.82 ) (see online supplementary table).

\section{DISCUSSION}

Our study shows that a high proportion (57\%) of patients with ACS in Europe and Latin America were receiving DAPT beyond 12 months, well before there was clear evidence of any 
Table 1 Antithrombotic medication at discharge in relation to characteristics of the study population

\begin{tabular}{|c|c|c|c|c|c|}
\hline \multirow[b]{2}{*}{ Description } & \multirow[b]{2}{*}{ Total number of patients in each category } & \multicolumn{3}{|c|}{$\begin{array}{l}\text { Distribution of antithrombotic medication at discharge by } \\
\text { characteristics of study population }\end{array}$} & \multirow[b]{2}{*}{ p Value } \\
\hline & & DAPT $(n=8593)$ & SAPT $(n=899)$ & Anticoagulants $(n=537)$ & \\
\hline Age, years; mean (SD) & & $61(12)$ & $64(12)$ & $67(13)$ & $<0.0001$ \\
\hline Group, years; n (\%) & & & & & $<0.0001$ \\
\hline$<50$ & 1691 & $1517(89.7)$ & $118(7.0)$ & $56(3.3)$ & \\
\hline $50-59$ & 2821 & $2523(89.4)$ & $206(7.3)$ & $92(3.3)$ & \\
\hline $60-69$ & 2794 & $2386(85.4)$ & $271(9.7)$ & $137(4.9)$ & \\
\hline $70-79$ & 1962 & $1558(79.4)$ & $226(11.5)$ & $178(9.1)$ & \\
\hline$\geq 80$ & 760 & $608(80.0)$ & $78(10.3)$ & $74(9.7)$ & \\
\hline Female, n (\%) & 2467 & $2011(81.5)$ & $295(11.9)$ & $161(6.5)$ & $<0.0001$ \\
\hline Region, $n(\%)$ & & & & & $<0.0001$ \\
\hline Northern Europe & 3526 & $3008(85.3)$ & $283(8.0)$ & $235(6.7)$ & \\
\hline Southern Europe & 2290 & $1958(89.4)$ & $135(6.2)$ & $97(4.4)$ & \\
\hline Eastern Europe & 2291 & 1954 (85.3) & $224(9.8)$ & $113(4.9)$ & \\
\hline Latin America & 2022 & $1673(82.7)$ & $257(12.7)$ & $92(4.6)$ & \\
\hline Centre type, n (\%) & & & & & 0.0002 \\
\hline University general & 3930 & $3370(85.8)$ & $362(9.2)$ & $198(5.0)$ & \\
\hline Non-university general & 2848 & $2487(87.3)$ & $212(7.4)$ & $149(5.2)$ & \\
\hline Regional/community/rural & 2137 & $1809(84.7)$ & $192(9.0)$ & $136(6.4)$ & \\
\hline Private & 1060 & $927(87.5)$ & $133(12.5)$ & $0(0)$ & \\
\hline Diabetic & 2256 & 1882 (83.4) & $227(10.1)$ & $147(6.5)$ & 0.002 \\
\hline ACS type, n (\%) & & & & & $<0.0001$ \\
\hline STEMI & 4747 & $4314(90.9)$ & $213(4.5)$ & $220(4.6)$ & \\
\hline NSTE-ACS & 5251 & $4253(81.0)$ & $681(13.0)$ & $317(6.0)$ & \\
\hline Management strategy, n (\%) & & & & & $<0.0001$ \\
\hline $\mathrm{PCl}$ only & 6572 & 6205 (94.4) & $110(1.7)$ & $257(3.9)$ & \\
\hline CABG & 258 & $50(19.4)$ & $179(69.4)$ & $29(11.2)$ & \\
\hline Medication only & 3145 & $2292(72.9)$ & $603(19.2)$ & $250(7.9)$ & \\
\hline Serum creatine, mg/dL; median (IQR) & 9470 & $0.90(0.79-1.08)$ & $0.93(0.80-1.11)$ & $1.00(0.83-1.20)$ & $<0.0001$ \\
\hline Blood glucose, mg/dL; median (IQR) & 8709 & $122.0(104-150)$ & $117.1(101-151)$ & $124.7(104-162)$ & $<0.0001$ \\
\hline Haemoglobin g/dL; median (IQR) & 9358 & $14.3(13.1-15.3)$ & $13.9(12.7-15.0)$ & $13.9(13.0-15.0)$ & 0.20 \\
\hline
\end{tabular}

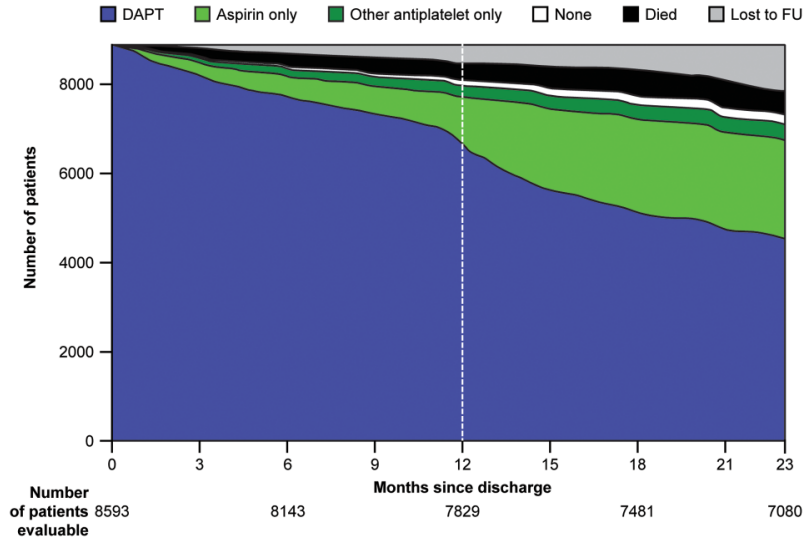

Figure 1 Changes in medication status during follow-up among patients with ACS discharged on DAPT. ACS, acute coronary syndrome; DAPT, dual antiplatelet therapy; FU, follow-up.

advantage of such practice or any recommendations by contemporary clinical practice guidelines. ${ }^{5}$ Interestingly, we could not find any strong patient-related determinant of longer DAPT duration, including initial revascularisation strategy, and use or type of stents. Conversely, the largest variations were found in individual country practices, suggesting local systems as more important drivers of local DAPT duration. Moreover, while the findings showed no overall correlation between switching from DAPT to a lower antiplatelet level treatment and CV events, they suggest an increased global CV risk within the first 7 days after treatment change with no further increase in coronary or mortality risk.

Currently, DAPT for longer than 12 months may be considered in patients after ACS, according to the most recent guidelines. ${ }^{13}$ However, this recommendation is based on findings from the DAPT and PEGASUS-TIMI 54 (Prevention of Cardiovascular Events in Patients with Prior Heart Attack Using Ticagrelor Compared to Placebo on a Background of AspirinThrombolysis in Myocardial Infarction 54) trials, ${ }^{1} 14$ published in December 2014 and March 2015, respectively, years after the treatment decisions were made. Although surprising, our results are not unique. The PARIS (Patterns of Non-Adherence to Antiplatelet Regimens in Stented Patients) registry reported that $43 \%$ of patients with ACS and $57 \%$ of those who underwent elective PCI remained on DAPT at the end of a 2-year follow-up in a cohort of 5031 patients from the USA, France, Germany, Italy and Greece. ${ }^{8}$ Similar results had been reported in France ${ }^{9}$ and the USA.? 
Table 2 Characteristics of patients staying on DAPT during all follow-up

\begin{tabular}{|c|c|c|c|c|}
\hline Characteristic & $\begin{array}{l}\text { Number }(\%) \text { of } \\
\text { patients remaining on } \\
\text { DAPT at } 12 \text { months }(n=6552)\end{array}$ & p Value* & $\begin{array}{l}\text { Number }(\%) \text { of } \\
\text { patients remaining on } \\
\text { DAPT at the end of follow-up }(n=4859)\end{array}$ & p Value* \\
\hline Age, yearst & & 0.55 & & 0.018 \\
\hline$<50$ & $1147 / 1517$ (75.6) & & $819 / 1517(54.0)$ & \\
\hline $51-59$ & 1949/2523 (77.2) & & $1393 / 2523(55.2)$ & \\
\hline $60-69$ & $1848 / 2386(77.5)$ & & $1352 / 2386(56.7)$ & \\
\hline $70-79$ & 1230/1558 (78.9) & & $916 / 1558(58.8)$ & \\
\hline$\geq 80$ & $464 / 608(76.3)$ & & $378 / 608(62.2)$ & \\
\hline Sex & & 0.59 & & 0.91 \\
\hline Male & $5072 / 6582(77.1)$ & & $3706 / 6582(56.3)$ & \\
\hline Female & $1567 / 2011(77.9)$ & & $1153 / 2011(57.3)$ & \\
\hline Region & & $<0.001$ & & $<0.001$ \\
\hline Northern Europe & $2467 / 3008(82.0)$ & & $1825 / 3008(60.7)$ & \\
\hline Southern Europe & 1495/1958 (76.4) & & 1005/1958 (51.3) & \\
\hline Eastern Europe & $1372 / 1954(70.2)$ & & $1002 / 1954(51.3)$ & \\
\hline Latin America & 1305/1673 (78.0) & & $1027 / 1673(61.4)$ & \\
\hline Centre type & & $<0.001$ & & $<0.001$ \\
\hline University general & $2540 / 3370(75.4)$ & & $1913 / 3370(56.8)$ & \\
\hline Non-university general & $1921 / 2487(77.2)$ & & $1335 / 2487(53.7)$ & \\
\hline Regional/community/rural & $1471 / 1809(81.3)$ & & $1118 / 1809(61.8)$ & \\
\hline Private & 707/927 (76.3) & & $493 / 927(53.2)$ & \\
\hline Type of ACS† & & 0.16 & & 0.68 \\
\hline NSTE-ACS & $3262 / 4253(76.7)$ & & $2430 / 4253(57.1)$ & \\
\hline STEMI & 3360/4314 (77.9) & & $2415 / 4314(56.0)$ & \\
\hline Diabetic status & & 0.034 & & 0.008 \\
\hline Non-diabetic & $5142 / 6711$ (76.6) & & $3725 / 6711(55.5)$ & \\
\hline Diabetic & $1497 / 1882(79.5)$ & & $1134 / 1882(60.3)$ & \\
\hline Management strategy $\dagger$ & & $<0.001$ & & 0.25 \\
\hline CABG (with/without PCI) & $40 / 50(80.0)$ & & $32 / 50(64.0)$ & \\
\hline $\mathrm{PCl}$ only & $1679 / 2292(73.3)$ & & $3466 / 6205(55.9)$ & \\
\hline Medication only & 1679/2292 (73.3) & & 1339/2292 (58.4) & \\
\hline Unknown & $29 / 46(63.0)$ & & $22 / 46(47.8)$ & \\
\hline Antiplatelet medication & & 0.99 & & $<0.001$ \\
\hline Aspirin+clopidogrel & $5420 / 7829(69.2)$ & & $4495 / 7829(57.4)$ & \\
\hline Aspirin+prasugrel & $500 / 705(70.9)$ & & $333 / 705(47.2)$ & \\
\hline Aspirin+clopidogrel+prasugrel & $14 / 18(77.8$ & & $10 / 18(55.6)$ & \\
\hline Aspirin+ticlopidine & $30 / 41(73.2)$ & & $21 / 41(51.2)$ & \\
\hline Glucose, mg/dLt & & 0.19 & & 0.061 \\
\hline$<160$ & $4381 / 5709(76.7)$ & & $3194 / 5709(55.9)$ & \\
\hline$\geq 160$ & 1390/1763 (78.8) & & 1056/1763 (59.9) & \\
\hline Creatine, mg/dL $\dagger$ & & 0.17 & & 0.88 \\
\hline$<1.2$ & $5455 / 7041(77.5)$ & & 3959/7041 (56.2) & \\
\hline $1.2-1.6$ & $549 / 713(77.0)$ & & $407 / 713(57.1)$ & \\
\hline $1.6-2.0$ & $154 / 200(77.0)$ & & $121 / 200(60.5)$ & \\
\hline$>2.0$ & 129/167 (77.2) & & $113 / 167(67.7)$ & \\
\hline Haemoglobin, g/dL $\dagger$ & & 0.68 & & 0.055 \\
\hline$<13$ & $1321 / 1683(78.5)$ & & $1020 / 1683(60.6)$ & \\
\hline$\geq 13$ & $4892 / 6357(77.0)$ & & $3512 / 6357(55.2)$ & \\
\hline
\end{tabular}

The reasons for such practices are unknown. In EPICOR, it is surprising that there were only small differences in DAPT patterns according to critical factors related to long-term risk, such as age, diabetes, initial strategy or type of stent. Neither were there any major differences among the four predefined regions in the proportion of patients on DAPT at the end of follow-up, ranging from $55.5 \%$ in southern Europe to $66 \%$ in Latin
America. However, there were major differences between countries within the same region. In fact, country represented the most important determinant of the proportion of patients remaining on DAPT at the end of follow-up. This suggests that duration of DAPT may be mostly conditioned by local factors rather than by an individual patient's risk. Whether these factors are cultural, economic or related to organisational factors within 


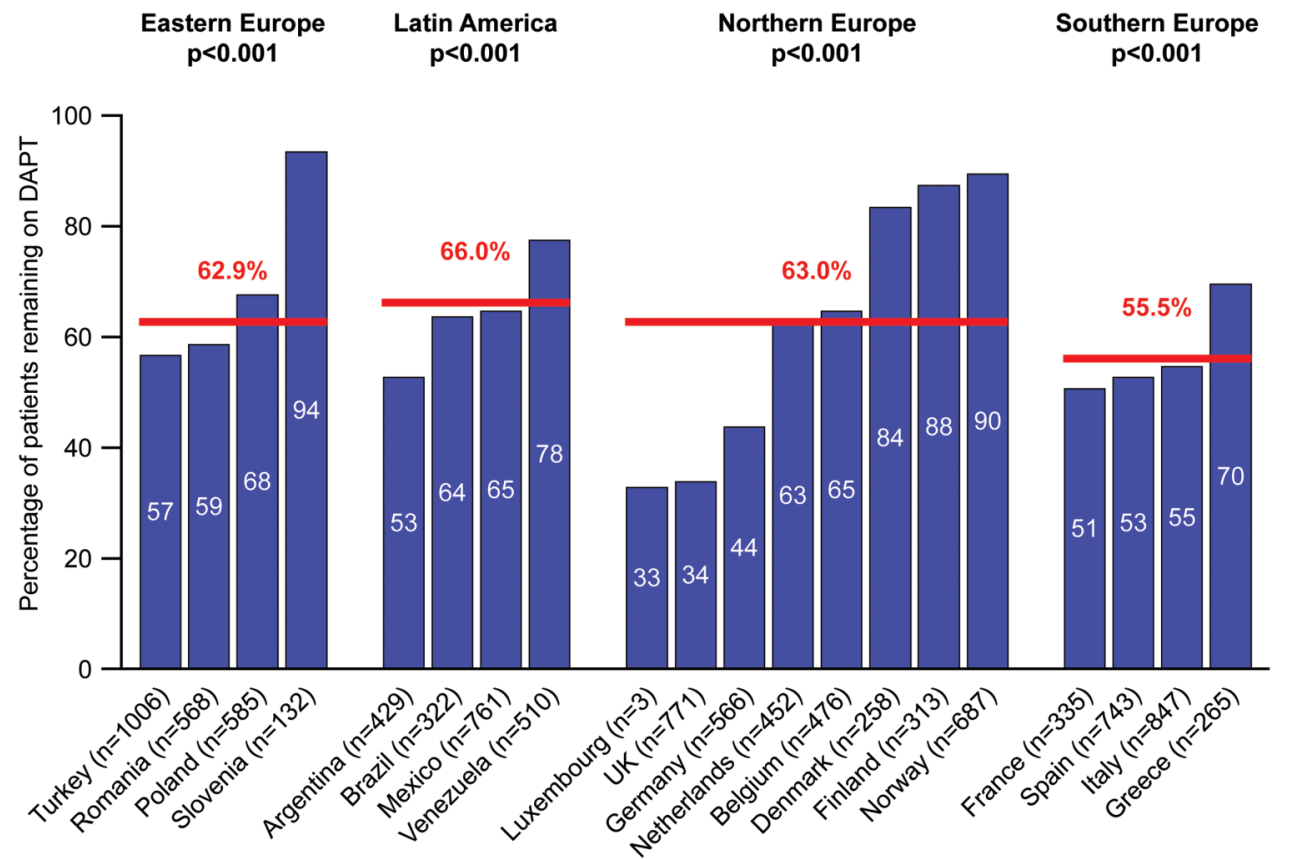

Figure 2 Proportion of patients on DAPT at the end of follow-up by country. This analysis excluded patients who died or were lost to follow-up. DAPT, dual antiplatelet therapy.

Table 3 Cardiovascular (CV) and bleeding events during follow-up in the whole cohort $(n=10069)$

\begin{tabular}{lrc}
\hline Type of event & $\begin{array}{c}\text { Patients with } \\
\text { event (\%)* }\end{array}$ & $\begin{array}{l}\text { Total number } \\
\text { of events (fatal) }\end{array}$ \\
\hline All CV & $978(9.8 \%)$ & $1173(281)$ \\
Coronary & $559(5.7 \%)$ & $653(65)$ \\
MI & $238(2.5 \%)$ & $252(45)$ \\
Heart failure & $104(1.1 \%)$ & $117(30)$ \\
Arrhythmia & $88(0.9 \%)$ & $93(13)$ \\
Sudden death & $8(0.1 \%)$ & $8(8)$ \\
Chest pain & $14(0.1 \%)$ & $18(0)$ \\
Cerebrovascular & $50(0.5 \%)$ & $56(8)$ \\
Other & $71(0.7 \%)$ & $75(7)$ \\
Not classified & $151(1.5 \%)$ & $153(150)$ \\
Bleeding & $366(3.6 \%)$ & $492(14)$ \\
Clinically relevant bleeds & $168(1.7 \%)$ & $223(14)$ \\
$\quad$ Bleeds requiring hospitalisation & $158(1.5 \%)$ & $212(8)$ \\
$\quad$ Bleeds with haemodynamic & $33(0.3 \%)$ & $37(3)$ \\
$\quad$ compromise & & \\
$\quad$ Intracranial bleeds & $15(0.2 \%)$ & $15(6)$ \\
Other bleeds & $226(2.2 \%)$ & $269(0)$ \\
Deaths not due to CV or bleeding & & \\
Deaths of unknown cause & $84(0.9 \%)$ & $84(84)$ \\
\hline
\end{tabular}

*Percentages are Kaplan-Meier estimates at 23 months. $\mathrm{MI}$, myocardial infarction.

local healthcare systems, requires further investigation. Given the recent results from the DAPT and PEGASUS-TIMI 54 trials, ${ }^{1}{ }^{14}$ extended DAPT use may increase even further in the future. Postmarketing surveillance at both a national and an international level will be needed to monitor patterns of DAPT duration and, particularly, patient selection for long-term DAPT duration.

In EPICOR, event rates continued to accrue throughout the 2-year follow-up period post discharge with higher rates in the initial period and a steady increase during subsequent months up to the end of follow-up. Interestingly, $85 \%$ of events and $85 \%$ of deaths during follow-up occurred while patients were on DAPT; approximately half of non-fatal events were coronary, and one in four patients who presented with an event died during follow-up. The incidence of clinically relevant bleeding recorded in EPICOR was relatively low (approximately 5\%), and less than half of the events were considered clinically relevant. The PARIS registry showed an incidence of TIMI major bleeding of $1.4 \%$ and $2.1 \%$ at 1 year and 2 years, respectively. ${ }^{8}$ Unfortunately, our findings cannot be compared here due to the absence of a standardised bleeding definition.

Our study shows an association between DAPT discontinuation (for any cause, including interruption or disruption) and adverse events in the first 7 days. The DAPT trial showed a modest increase in event rate between month 30, when the $\mathrm{P}_{2} \mathrm{Y}_{12}$ inhibitor was terminated, and month 33 when follow-up was terminated, ${ }^{1}$ suggesting a potential 'rebound' effect after DAPT discontinuation, which was more obvious after prasugrel withdrawal. ${ }^{15}$ The real-life PARIS registry also reported an increased risk of major adverse CV events, spontaneous MI, definite or probable stent thrombosis and cardiac death after DAPT cessation, which was particularly relevant within the first 7 days. ${ }^{8}$ However, this hazard was only observed for DAPT disruptions; that is, cessations due to bleeding or patient nonadherence, while DAPT discontinuations (those recommended by physicians for patients thought to no longer need DAPT) or interruptions (temporary cessations due to surgical necessity with reinstitution of DAPT within 14 days) were not associated with an increased event rate. It is worth noting that our definition of DAPT discontinuation does not align exactly with the classification used in PARIS, which may explain at least part of the differences in magnitude of effect. Moreover, despite the large number of patients enrolled in EPICOR, the number who effectively dropped DAPT was not large and the absolute number of events recorded was low, limiting the power for accurate risk estimations. Results for longer-term DAPT duration 


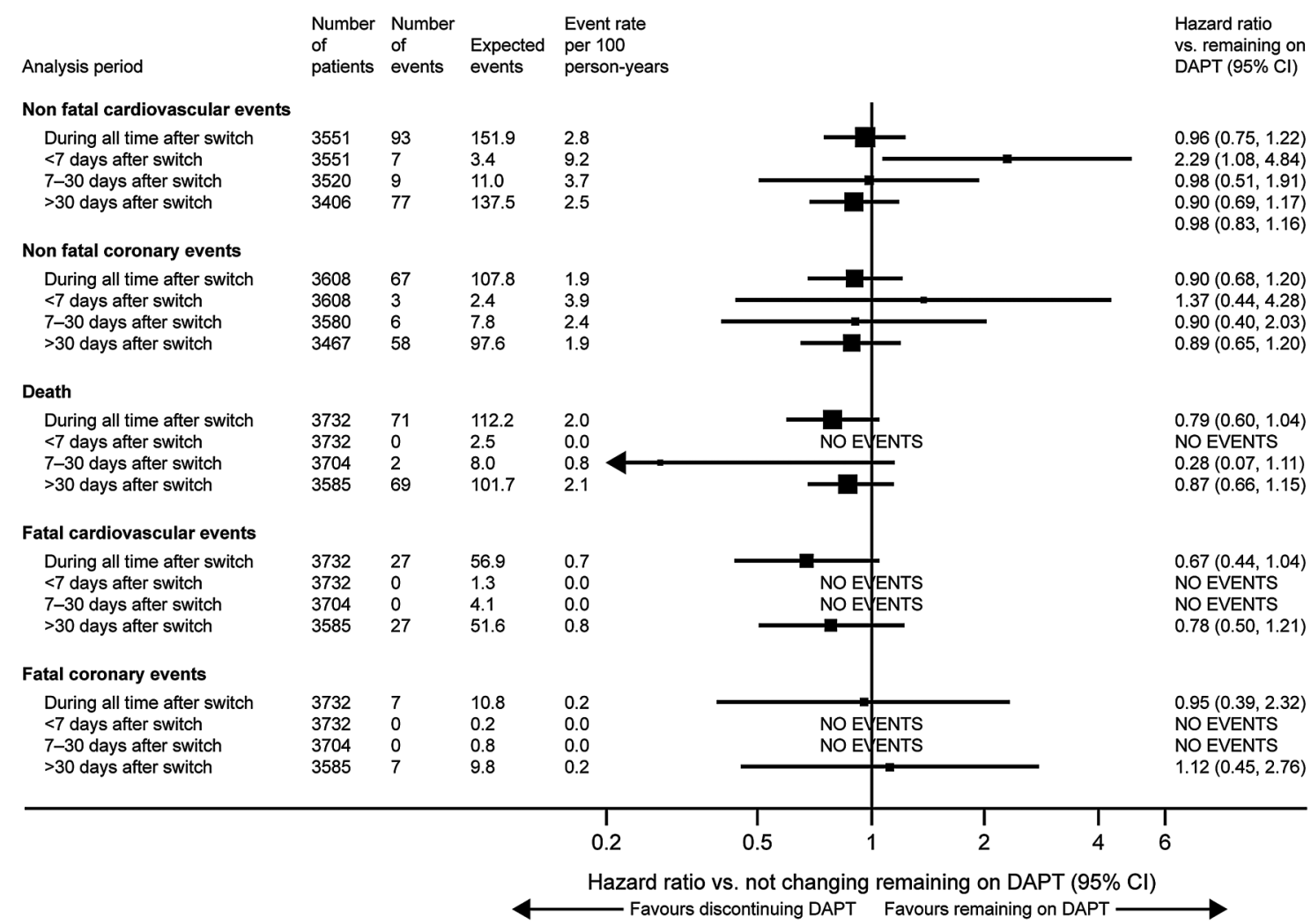

Figure 3 Risk of CV or coronary events following interruption of DAPT versus remaining on DAPT. CV, cardiovascular; DAPT, dual antiplatelet therapy.

are unknown as such trials are not available. The French registry of Acute ST elevation or non-ST-elevation Myocardial Infarction 2005 registry, however, reported similar outcomes in patients with or without prolonged DAPT in the long term (up to 5 years of follow-up). ${ }^{9}$

The patterns found in our registry are more surprising in the light of the later evidence, coming mostly from the results of the two large randomised trials comparing either 12 months versus 30 months of DAPT with aspirin and clopidogrel or prasugrel in patients who had received a coronary stent (DAPT trial), ${ }^{1}$ or aspirin versus aspirin plus ticagrelor starting between 12 months and 36 months after a prior MI (either continuing DAPT at 12 months or restarting DAPT later) (PEGASUS-TIMI 54 trial). ${ }^{14}$ Both trials showed a reduction in ischaemic events (MI in DAPT, and MI and stroke in PEGASUS-TIMI 54) with a significant and clinically relevant increase in major bleeding and diverging but non-significant trends for mortality. Together with a number of smaller trials testing different durations of DAPT, mostly in patients treated with stents, several meta-analyses have confirmed that prolongation of DAPT beyond the first 12 months is a trade-off between reducing non-fatal ischaemic events and non-fatal major bleeding events, with unclear results on all-cause mortality, which may differ according to the reason for DAPT indication (MI vs percutaneous intervention). ${ }^{16-18}$ For this reason, the 2015 European Society of Cardiology guidelines for the management of ACS in patients presenting without persistent ST segment elevation state that $\mathrm{P}_{2} \mathrm{Y}_{12}$ inhibitor administration in addition to aspirin beyond 1 year may be considered after careful assessment of the ischaemic and bleeding risks of the patient (indication class, IIb; level of evidence A). ${ }^{12}$

Several limitations should be considered when interpreting the results of this study. As for any observational study, causal relationships between patterns of care or DAPT interruption and outcomes cannot be inferred. Some methodological issues may have affected the accuracy of data collection or evaluation. There was no prescription ascertainment and no confirmation with supervising physician, so it may be possible that telephone call answers do not detail actual tablets prescribed or taken. Given the fact that telephone calls were only made every 3 months, recall bias leading to a reduced number of events reported cannot be excluded. There is also a possibility that some patients might have interpreted the telephone call as a reminder to take the medication and could have felt tempted to respond they were taking their medication, potentially leading to an artefact in the direction of longer medication use. This is particularly relevant for fatal events in which failure to identify previous changes in medication may have been an issue that could affect calculations of estimated risk. CV event rates may also have been underestimated as no central adjudication of events was done and only those events confirmed by physicians with full event reports were counted in the analyses. Local adjudication might have been open to potentially differing interpretations in different countries. Furthermore, cause of death was not systematically recorded, and clinical events during follow-up were not centrally adjudicated. Also, there was no standardised classification of clinically relevant bleeding, limiting the comparability of our results. Finally, there were ongoing changes in prescription patterns of antiplatelet drugs during the study period.

\section{CONCLUSIONS}

International patterns of DAPT duration do not necessarily align with guideline recommendations. Despite contemporary evidence, patients with ACS remained on DAPT longer than 12 months, irrespective of clinical characteristics and ischaemic 
risk, country being the most important determinant of DAPT duration. This emphasises the importance of postmarketing surveillance to monitor prescription patterns, patient selection and clinical events related to long-term DAPT duration.

\section{Key messages}

\section{What is already known on this subject?}

Although guidelines recommend 12 months of DAPT following an acute coronary syndrome (ACS) in the majority of patients, relatively little is known about the duration of DAPT in a real-world setting.

\section{What might this study add?}

This large international, prospective study shows that many patients with ACS remain on DAPT beyond 12 months. There were large differences in DAPT duration between countries, but only small differences according to patient characteristics, therapeutic strategy or region, suggesting local policies rather than patient characteristics as key determinants of DAPT prescription patterns. Discontinuation of DAPT was associated with a short-term increase in overall cardiovascular risk but not with any long-term increase in coronary events or mortality.

\section{How might this impact on clinical practice?}

The findings suggest that long-term duration of DAPT after ACS was already common practice before it was recommended. There is a need to follow compliance with guidelines recommendations and develop interventions to improve these.

Acknowledgements The authors thank Liz Anfield, Prime Medica Ltd, for medical writing support, Worldwide Clinical Trials for data management, and all the EPICOR principal investigators.

Contributors All authors contributed to the design and conduct of the study, analysis of the study data, and opinions, conclusions and interpretation of the data. The lead author, HB, takes responsibility for the initial drafting and overall content of the manuscript, and all authors contributed to the manuscript at each stage of preparation, and approved the final version. Statistical analysis was performed by JG and SP.

Funding The EPICOR study was funded by AstraZeneca.

Competing interests HB has received advisory/consulting fees from Abbott, AstraZeneca, Bayer, BMS-Pfizer, Daichii-Sankyo, Eli-Lilly, Ferrer, Menarini, Novartis, Sanofi and Servier, and research grants from AstraZeneca. SP has received research funding from AstraZeneca. ND has received research grants from Amgen, AstraZeneca, Bayer, Daiichi-Sankyo, Eli-Lilly, MSD and Sanofi, and consulting or speaking fees from Amgen, AstraZeneca, Bayer, BMS, Boehringer-Ingelheim, GSK, MSD-Schering Plough, Novartis, Pierre Fabre, Pfizer, Roche, Sanofi-Aventis, Servier and The Medicines Company. LA has received consulting and lecture fees from AstraZeneca. JM is an employee of AstraZeneca. JG has received research funding from AstraZeneca. FVdW has received consulting fees and research grants from Boehringer-Ingelheim and Merck, and consulting fees from Roche, Sanofi-Aventis, AstraZeneca and The Medicines Company.

Ethics approval Local/national ethics committees for each participating centre/ country.
Provenance and peer review Not commissioned; externally peer reviewed.

Open Access This is an Open Access article distributed in accordance with the Creative Commons Attribution Non Commercial (CC BY-NC 4.0) license, which permits others to distribute, remix, adapt, build upon this work non-commercially, and license their derivative works on different terms, provided the original work is properly cited and the use is non-commercial. See: http://creativecommons.org/ licenses/by-nc/4.0/

\section{REFERENCES}

1 Mauri L, Kereiakes DJ, Yeh RW, et al. Twelve or 30 months of dual antiplatelet therapy after drug-eluting stents. N Engl J Med 2014;371:2155-66.

2 Yusuf S, Zhao F, Mehta SR, et al. Effects of clopidogrel in addition to aspirin in patients with acute coronary syndromes without ST-segment elevation. N Engl J Med 2001;345:494-502.

3 Wiviott SD, Braunwald E, McCabe $\mathrm{CH}$, et al. Prasugrel versus clopidogrel in patients with acute coronary syndromes. N Engl J Med 2007;357:2001-15.

4 Wallentin L, Becker RC, Budaj A, et al. Ticagrelor versus clopidogrel in patients with acute coronary syndromes. N Engl J Med 2009;361:1045-57.

5 Hamm CW, Bassand JP, Agewall S, et al. ESC Guidelines for the management of acute coronary syndromes in patients presenting without persistent ST-segment elevation: The Task Force for the management of acute coronary syndromes (ACS) in patients presenting without persistent ST-segment elevation of the European Society of Cardiology (ESC). Eur Heart J 2011;32:2999-3054.

6 Steg PG, James SK, Atar D, et al. ESC Guidelines for the management of acute myocardial infarction in patients presenting with ST-segment elevation. Eur Heart J 2012;33:2569-619.

7 Mulukutla SR, Marroquin OC, Vlachos HA, et al. Benefit of long-term dual anti-platelet therapy in patients treated with drug-eluting stents: from the $\mathrm{NHLBI}$ dynamic registry. Am J Cardiol 2013:111:486-92.

8 Mehran R, Baber U, Steg PG, et al. Cessation of dual antiplatelet treatment and cardiac events after percutaneous coronary intervention (PARIS): 2 year results from a prospective observational study. Lancet 2013;382:1714-22.

9 Schiele F, Puymirat E, Bonello L, et al. Impact of prolonged dual antiplatelet therapy after acute myocardial infarction on 5-year mortality in the FAST-MI 2005 registry. Int J Cardiol 2015;187:354-60.

10 Bueno $\mathrm{H}$, Danchin N, Tafalla M, et al. EPICOR (long-tErm follow-up of antithrombotic management Patterns In acute CORonary syndrome patients) study: rationale, design, and baseline characteristics. Am Heart J 2013;165:8-14.

11 Bueno $H$, Sinnaeve $P$, Annemans $L$, et al. Opportunities for improvement in anti-thrombotic therapy and other strategies for the management of acute coronary syndromes: Insights from EPICOR, an international study of current practice patterns. Eur Heart J Acute Cardiovasc Care 2016;5:3-12.

12 Altman DG, De Stavola BL. Practical problems in fitting a proportional hazards model to data with updated measurements of the covariates. Stat Med 1994:13:301-41.

13 Roffi M, Patrono C, Collet JP, et al. 2015 ESC Guidelines for the management of acute coronary syndromes in patients presenting without persistent ST-segment elevation: Task Force for the Management of Acute Coronary Syndromes in Patients Presenting without Persistent ST-Segment Elevation of the European Society of Cardiology (ESC). Eur Heart J 2016;37:267-315.

14 Bonaca MP, Bhatt $\mathrm{DL}$, Cohen $\mathrm{M}$, et al. Long-term use of ticagrelor in patients with prior myocardial infarction. N Engl J Med 2015;372:1791-800.

15 Garratt KN, Weaver WD, Jenkins RG, et al. Prasugrel plus aspirin beyond 12 months is associated with improved outcomes after taxus liberte Paclitaxel-eluting coronary stent placement. Circulation 2015;131:62-73.

16 Palmerini T, Benedetto U, Bacchi-Reggiani L, et al. Mortality in patients treated with extended duration dual antiplatelet therapy after drug-eluting stent implantation: a pairwise and Bayesian network meta-analysis of randomised trials. Lancet 2015;385:2371-82.

17 Navarese EP, Andreotti F, Schulze V, et al. Optimal duration of dual antiplatelet therapy after percutaneous coronary intervention with drug eluting stents: meta-analysis of randomised controlled trials. BMJ 2015:350:h1618

18 Udell JA, Bonaca MP, Collet JP, et al. Long-term dual antiplatelet therapy for secondary prevention of cardiovascular events in the subgroup of patients with previous myocardial infarction: a collaborative meta-analysis of randomized trials. Eur Heart J 2016:37:390-9. 